\title{
Implementing and managing community-based education and service learning in undergraduate health sciences programmes: Students' perspectives
}

\author{
S B Kruger, ${ }^{1} \mathrm{PhD} ; \mathbf{M} \mathbf{M}$ Nel, ${ }^{1} \mathrm{PhD} ; \mathbf{G} \mathbf{J}$ van $\mathrm{Zyl},{ }^{2} \mathrm{MB} \mathrm{ChB}, \mathrm{MMed}, \mathrm{PhD}$ \\ ${ }^{1}$ Division of Health Sciences Education, Office of the Dean, Faculty of Health Sciences, University of the Free State, Bloemfontein, South Africa \\ ${ }^{2}$ Office of the Dean, Faculty of Health Sciences, University of the Free State, Bloemfontein, South Africa
}

Corresponding author: S B Kruger (krugersb@ufs.ac.za)

Background. A current challenge in the training of healthcare professionals is to produce socially responsive graduates who are prepared for work in community settings. Community-based education (CBE) and service learning (SL) are teaching approaches used in the Faculty of Health Sciences at the University of the Free State (UFS), Bloemfontein, South Africa to address these challenges. Students have different views with regard to CBE and SL, and by surveying these perceptions information can be gained on how to better integrate CBE and SL into learning programmes.

Objective. To investigate students' perceptions of CBE and SL in a health sciences faculty.

Methods. Nominal group discussion was conducted to identify topics to explore students' perceptions of CBE and SL. A questionnaire was administered to all undergraduate health sciences students at UFS to survey their perceptions of CBE and SL.

Results. Twenty different themes were identified that had a positive or negative impact on the students' perceptions of CBE and SL. Positive aspects included personal growth, exposure to a diversity of patients, gaining practical experience and enhancement of inter- and intrapersonal skills. However, the students perceived the following as negative or inadequate: the organisation of CBE and SL; availability of resources; attitude of healthcare professionals; and prior orientation.

Conclusion. CBE and SL need to be carefully implemented and managed to enhance the learning experience for students and produce socially responsive healthcare professionals who are equipped to address the healthcare challenges in their communities.

Afr J Health Professions Educ 2015;7(2):161-164. DOI:10.7196/AJHPE.333

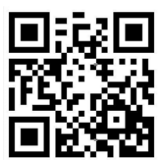

Globally, more than a billion people never consult a healthcare worker in their lives. Inequitable and ineffective healthcare systems are weakened by a scarcity and maldistribution of the healthcare workforce. ${ }^{[1]}$ Together with other stakeholders, health professional schools can play a key role in reducing inequality and improving health equity. The Global Independent Commission on Education of Health Professionals for the 21st Century (2010) calls for transforming institutional and educational approaches to better meet changing health systems needs. ${ }^{[2]}$ Furthermore, in late 2010, the Global Consensus for Social Accountability of Medical Schools (GCSA) urged schools to improve their response to current and future health-related needs and challenges in society and reorientate their activities accordingly. ${ }^{[3]}$ From the above it follows that it is necessary for health professional schools to engage with the community as an essential strategy to achieve a diverse healthforce, increasing access to healthcare and eliminating health disparities.

In South Africa (SA), as in the rest of the world, community engagement plays an important part in higher education. Reorientation of health professions education to an inclusive primary healthcare approach was called for in the White Paper on the Transformation of the Healthcare System in SA. ${ }^{[4]}$ Some ${ }^{[5,6]}$ argue that the educational programme for healthcare professionals should deliver graduates who are prepared for work in community settings, resulting from the move from fixed institutions, such as hospitals, to various settings in the community. Community-based education (CBE) and service learning (SL) as a means of achieving greater social responsibility have become more prominent in health professions education worldwide.
CBE in a medical context can be defined as learning activities that take place within communities and take into consideration the main health problems of the country, but do not directly engage the community in the design, conduct and/or evaluation of these activities. ${ }^{[7,8]}$

SL has been defined as 'an educational approach involving curriculum-based, credit-bearing learning experiences in which students (a) participate in contextualised, well-structured and organised service activities aimed at addressing identified service needs in a community, and (b) reflect on the service experiences in order to gain a deeper understanding of the linkage between curriculum content and community dynamics, as well as achieve personal growth and a sense of social responsibility. It requires a collaborative partnership context that enhances mutual, reciprocal teaching and learning among all members of the partnership. ${ }^{[9]}$

The Faculty of Health Sciences at the University of the Free State (UFS), Bloemfontein, SA recognises the tremendous potential of CBE and SL to enhance health professions education, as both allow students to apply the information they learn in the classroom to real-world settings and provide an important avenue for self-reflection. CBE and SL contributed to the restructuring of clinical education in the Faculty, and various modules using CBE and SL are offered throughout the different study years across the various programmes. In our Faculty, CBE and SL vary, e.g. in terms of duration and number of outreaches.

Students' ability to learn is to a great extent affected by their perception of the specific learning environment. Perception in this study refers to the way students view, understand and interpret CBE and SL. If students have a 
negative perception of the learning environment, they will have a negative attitude towards the learning that is expected to take place, which could result in less effort put into the task at hand and ultimately less learning. Positive attitudes and perceptions may be encouraged by creating a better learning climate, ensuring the quality and quantity of the resources and gaining individual acceptance of the students. ${ }^{[10]}$

It is important to understand students' perceptions with regard to $\mathrm{CBE}$ and SL and take these into consideration in the design and implementation to enhance the students' experiences, which will in turn enhance the success thereof and result in graduates who are more socially responsive, able and willing to work in community settings.

\section{Methods}

\section{Study design}

A combination of methods was used to generate data; these findings were interpreted to form the basis for the recommendations on the improvement of CBE and SL. During Phase I of the mixed-methods research design, data were collected by means of a nominal group technique. Nominal group discussions were held with the class leaders to identify possible themes/ topics to describe the perceptions of health sciences students with regard to CBE and SL. These themes and topics were used to design a questionnaire, which formed Phase II of the research. ${ }^{[1]}$

The questionnaire survey used a quantitative approach with elements of qualitative research. The aim of the survey was to obtain demographic information of the participants, explore the students' perceptions regarding CBE and SL, and identify whether there are certain factors that influence students' experiences of $\mathrm{CBE}$ and SL.

The questionnaire contained both open-ended and closed questions, with various scaling methods, e.g. nominal measurements were used in the demography section, while a Likert scale was used to obtain measurements from the closed questions in the other sections. ${ }^{[11]}$ Sufficient open space was provided after the open-ended questions so that respondents could write down their comments/responses.

\section{Study population}

A questionnaire survey was administered to all students registered for undergraduate degree programmes in the Faculty of Health Sciences, UFS, who participated in CBE and/or SL during 2011. Of the 1063 students registered, $792(74.5 \%)$ completed the survey (Table 1). The population

Table 1. Numbers of registered undergraduate students in the Faculty of Health Sciences, UFS, who completed the questionnaire survey $(N=792)$

\begin{tabular}{lllllll}
\hline & \multicolumn{5}{c}{ Year of study } & \\
\cline { 2 - 6 } Academic programmes & I & II & III & IV & V & Total \\
\hline MB ChB & 128 & $0^{*}$ & 99 & 54 & 76 & 357 \\
B Occupational Therapy & 31 & 31 & 6 & 27 & NA & 95 \\
BSc Physiotherapy & 40 & 33 & 38 & 30 & NA & 141 \\
BSc Dietetics & $0^{*}$ & $0^{*}$ & $0^{*}$ & 18 & NA & 18 \\
B Optometry & $0^{*}$ & $0^{*}$ & 23 & $0^{*}$ & NA & 23 \\
BSocSc (Nursing) & 60 & 27 & 30 & 41 & NA & 158 \\
$\quad$ Total & 259 & 91 & 196 & 170 & 76 & 792 \\
$\begin{array}{l}\text { NA }=\text { not applicable, as these programmes are 4-year degree courses. } \\
\text { * No CBE/SL in these study years. }\end{array}$ & \multicolumn{7}{c}{} & & & & &
\end{tabular}

included students instructed in either Afrikaans or English, as well as male and female students.

\section{Data collection}

Questionnaires were handed out to students directly after an academic contact session, following their completion of the CBE or SL section in their respective modules. Participation was voluntary and questionnaires were completed anonymously. Students had 20 minutes to complete the questionnaire, which gave them adequate time to consider their answers. After completion of the questionnaires, the participants placed these in a box to ensure anonymity and confidentiality.

\section{Data analysis}

Quantitative data from the questionnaire surveys were analysed descriptively by the Department of Biostatistics, UFS, using frequencies and percentages for the categorical variables. The qualitative data were analysed by the researcher (SBK) by reading and reflection, identification of themes, establishment of patterns and connections, as well as coding. Following the analysis of the data, both the quantitative and qualitative data were categorised into different themes.

Ethical approval was obtained from the Ethics Committee at the Faculty of Health Sciences, UFS (ECUFS No. 77/2011).

\section{Results}

Twenty different themes were identified and divided into aspects that had either a positive or a negative influence on the way students perceived CBE and/or SL. The themes are presented in Table 2, together with some of the verbatim quotes from the students to substantiate the theme.

\section{Discussion}

Based on the information obtained by means of the questionnaire survey, it was clear that the students regarded CBE and SL as valuable and enjoyed it. They agreed that by means of CBE and SL they had the opportunity to experience the 'human aspect' of patients, could put their knowledge into practice, and learn about the roles and values of the multidisciplinary teams. CBE and SL improved their sensitivity towards other cultures, self-confidence, interpersonal communication skills, problem-solving skills and other professional competencies.

However, a number of aspects that students perceived as negative were identified. Consequently, certain recommendations could be made with regard to the improvement of CBE and SL in the Faculty of Health Sciences, with a view to enhance the experiences of undergraduate health sciences students.

The researcher proposes that when implementing and managing CBE and SL in undergraduate health sciences programmes, certain steps should be included to enhance the students' experiences thereof and meet the underlying principles for which CBE and SL are intended (Kruger SB. Community based education and service learning: Experiences of health sciences students at the University of the Free State. Unpublished Doctoral Thesis. Bloemfontein: University of the Free State, 2013). The steps proposed by SBK concur with those set out by the Higher Education Quality Committee (HEQC) ${ }^{[11]}$ for the development of a curriculum model for service learning, with certain key steps in the development of a CBE programme. ${ }^{[12]}$

\section{Planning CBE and SL}

A number of important steps need to be taken when planning CBE and SL. The first step is to set clear module outcomes. It is crucial to ensure 


\section{Research}

Table 2. Themes that influenced students' perceptions of CBE and SL

\begin{tabular}{|c|c|}
\hline Positive & Verbatim quotes by students \\
\hline Achievement of personal growth & $\begin{array}{l}\text { 'It helped me to grow as a person which made me more competent in my personal life.' } \\
\text { 'Gained personal growth and development.' }\end{array}$ \\
\hline $\begin{array}{l}\text { Exposure to a variety of conditions/cases in } \\
\text { their respective fields of study }\end{array}$ & $\begin{array}{l}\text { 'Diversity in the patient profiles.' } \\
\text { 'It is challenging, there is a huge variety in patients.' }\end{array}$ \\
\hline Enhancement of social responsiveness & $\begin{array}{l}\text { 'There it feels like I am actually making a difference.' } \\
\text { 'It gives one a great sense of purpose.' }\end{array}$ \\
\hline Gaining interpersonal skills & $\begin{array}{l}\text { 'To enhance personal relations with my peers and other professionals.' } \\
\text { 'To be able to learn and experience with my fellow students.' }\end{array}$ \\
\hline Exposure to different cultures & $\begin{array}{l}\text { 'Working with different cultures.' } \\
\text { 'Give insight into other cultures.' }\end{array}$ \\
\hline Application of theoretical knowledge & $\begin{array}{l}\text { 'It is nice to experience everything in practical and not only to learn in theory.' } \\
\text { 'Challenging. Learn a lot more through experience.' }\end{array}$ \\
\hline Expanding theoretical knowledge & 'It gave me a new perspective and challenged me to search for solutions to some problems.' \\
\hline Gaining professional competencies & $\begin{array}{l}\text { 'Took me out of my comfort zone and I learnt new competencies.' } \\
\text { 'Had to make decisions yourself and bear responsibility. I enjoyed it.' }\end{array}$ \\
\hline Feeling valued in the community & $\begin{array}{l}\text { 'To see how thankful the people are afterwards.' } \\
\text { 'It was nice to see how thankful the less fortunate was for the help they receive.' }\end{array}$ \\
\hline Working in multidisciplinary teams & $\begin{array}{l}\text { 'To see how the multidisciplinary team works and experiences the hospital environment.' } \\
\text { 'It helps me to learn more about other fields.' }\end{array}$ \\
\hline $\begin{array}{l}\text { Gradual introduction into clinical field } \\
\text { Negative }\end{array}$ & 'It gives one a viewpoint of how things in the future in your career can work from early on in your studies.' \\
\hline Poor organisation & $\begin{array}{l}\text { 'To have set out schedules/time tables to minimize wastages.' } \\
\text { 'Place the students where there are learning opportunities other than being at a place yet you have nothing to do.' } \\
\text { 'Monitor students attendance regularly, it is bad that some students never attend sessions and lie and get away } \\
\text { with that.' }\end{array}$ \\
\hline Negative attitude of healthcare professionals & $\begin{array}{l}\text { 'The staff at the community centers should be encouraged to be more willing to teach.' } \\
\text { 'Only staff that are willing to be involved with students should be involved.' }\end{array}$ \\
\hline Feeling unproductive & $\begin{array}{l}\text { 'Place the students where there are learning opportunities.' } \\
\text { 'Theory in class does not match the practical aspects.' }\end{array}$ \\
\hline Exposure to traumatic situations & $\begin{array}{l}\text { 'The necessary debriefing after traumatic experiences.' } \\
\text { 'It gets overwhelming in the final year and there is very little support for the students, it gets very depressing at times.' }\end{array}$ \\
\hline Availability of resources & $\begin{array}{l}\text { 'Improve hospital conditions.' } \\
\text { 'The clinics need proper equipment.' }\end{array}$ \\
\hline Communication difficulties & $\begin{array}{l}\text { 'Communication between the faculty and the skills providers at the community facilities needs to be improved.' } \\
\text { 'An effort to communicate better with students as to the state of affairs.' } \\
\text { 'Learn Sesotho.' }\end{array}$ \\
\hline Transport problems & $\begin{array}{l}\text { 'Transport must be available.' } \\
\text { 'Not all students have cars, organise transport.' }\end{array}$ \\
\hline Insufficient orientation & $\begin{array}{l}\text { 'Enough training beforehand should be conducted.' } \\
\text { 'Maybe prepare students in depth as to what is expected of them.' }\end{array}$ \\
\hline Unstructured reflection & 'Reflections need to be more structured and done in private.' \\
\hline
\end{tabular}

that these outcomes address the healthcare needs of the community; clarify social, economic, cultural and political issues underlying the source of community needs; and enhance academic learning, personal growth and social responsibility of students. These outcomes should be clear, action orientated and measurable.

The second step is to assign tasks to the learning outcomes that are applicable to the students' level of knowledge and skills, in line with the outcomes and achievable in the community.
Thirdly, teaching methods can then be assigned to the different tasks. These teaching methods should be in the correct combination and level of students' knowledge and skills to facilitate and support learning from communities and integration of experiential learning.

The fourth and fifth steps are to assign assessment criteria and academic credits to the activities.

The next steps may be done in any order or simultaneously. Appropriate community service placements should be selected, with healthcare needs 
that relate to the set module outcomes, and students should have the appropriate skills and knowledge to address these needs. Furthermore, community settings should provide students with exposure to a variety of pathologies, be able to accommodate the students and have the necessary resources.

Academic staff should plan the duration of CBE and SL activities and schedule them in the students' academic rosters. Time frames should be reasonable to successfully complete the activities. Healthcare personnel from multiple professions and across disciplines have to be recruited and appointed. They should be knowledgeable, willing and able to supervise students and receive training with regard to their roles, responsibilities and module outcomes. Sufficient transport should be arranged and a professional counsellor or psychologist should be available to assist students with traumatic emotional experiences.

The final step that needs to be taken when planning CBE and SL is to orientate the students. Orientation should include the following elements:

- Introduce the concepts of CBE and SL.

- Emphasise the importance of CBE and SL.

- Prepare students with regard to what to expect.

- Orientate students to module-specific content.

- Explain what is expected of students in terms of outcomes.

- Explain assessment criteria.

- Discuss student reflections and indicate the frequency, format and content.

- Inform students about support services, e.g. transport, psychologist.

- Divide students into groups (multicultural and different languages).

- Hand out schedules.

- Provide clear directions to community sites.

- Inform students about professional courtesy, ethics, rights and responsibilities.

- Discuss the different activities that students need to perform.

- Discuss possible challenges and problems that students may encounter and possible solutions.

\section{Actions during $\mathrm{CBE}$ and SL}

The following actions should be executed on a continuous basis throughout the duration of CBE and SL: students' attendance should be strictly monitored at all times. Regular communication should take place between all stakeholders, including initial consultations with individuals at community settings and healthcare facilities at the beginning of CBE and SL to identify the broad healthcare needs of the community, negotiate CBE and SL activities and the aims and objectives of such activities, highlight the potential benefits for the community, and emphasise the importance of mutual respect and co-operation.

Other important actions are that academic staff should have regular meetings throughout the duration of CBE and SL at community settings and healthcare facilities with individuals, supervisors and students to ensure that everyone is working towards the same goal and objectives, confirm schedules for future CBE and SL, identify problems and challenges and discuss possible solutions. It should also be confirmed whether the necessary resources are available at the different sites where CBE and SL are planned.

\section{After CBE and SL}

On completion of CBE and SL it is important to celebrate the success with all the relevant role players and show appreciation for their respective inputs in the success of the initiative.

Allow students to evaluate CBE and SL and receive their inputs and feelings with regard to the experience. Evaluate whether module outcomes have been met and students' personal growth and social responsiveness have increased. These evaluations and recommendations may be used to adapt CBE and SL if necessary.

\section{Conclusion}

The current challenge of health professionals' training is that programmes should produce graduates who are prepared and willing to work in community settings to improve the current health status of individuals and groups and the health systems performance of the country in which they live. CBE and SL are teaching approaches to address this challenge. If CBE and SL could be implemented and managed successfully and students perceived it as positive learning experiences, they would acquire an understanding of the current health needs and challenges facing communities, have the opportunity to apply their theoretical knowledge, and learn in an environment that resembles their future professional career environment. Moreover, they will be equipped with a number of professional competencies and skills, experience interprofessional and multidisciplinary teams working together and interact with different cultures, while their social responsiveness will be enhanced.

The description of the perceptions of undergraduate health sciences students concerning CBE and SL generated from this study may be generalised beyond its local application as it may be applicable to other situations and groups. Therefore, the researcher proposes expansion of the use of the survey instrument developed for the purpose of this study by other faculties at UFS and other faculties of health sciences, both locally and internationally, as a means of generating comprehensive information regarding students' perceptions of CBE and SL in other settings, as well as encouraging future research in this regard.

\section{References}

1. The Training of Health Equity Network. THEnet's Social Accountability Evaluation Framework Version 1. Monograph I. 1st ed. New York: THEnet, 2011

2. Frenk J, Chen L, Bhutta ZA, et al. Health professionals for a new century: Transforming education to strengthen Frenk J, Chen L, Bhutta ZA, et al. Health professionals for a new century: Transforming education to strengthen
health systems in an interdependent world. Lancet 2010;376(9756):1923-1958. [http://dx.doi.org/10.1016/S01406736(10)61854-5]

3. Global Consensus for Social Accountability of Medical Schools: Position Statements Global Consensus for Socia Accountability of Medical Schools, 2010. http://healthsocialaccountability.org/ (accessed 10 January 2013). 4. Department of Education. A Programme for the Transformation of Higher Education. Education White Paper 3. Notice 1196. Pretoria: Government Printer, 1997.

5. Frank B, Adams MH, Edelstein J, Speakman E, Shelton M. Community-based nursing education of prelicensure students: Setting and supervision. Nurs Educ Perspect 2005;26(5):283-286.

6. Nokes K, Nickitas D, Keida R, Neville S. Does service-learning increase cultural competency, critical thinking and civic engagement? J Nurs Educ 2005;44(2):44-53.

7. Strasser R. Community engagement: A key to successful rural clinical education. Rural Remote Health 2010;10(3):1543.

8. Kristina TN, Majoor GD, van der Vleuten CP. Defining generic objectives for community-based education in undergraduate medical programmes. Med Educ 2004;38(5):510-521. [http://dx.doi.org/10.1046/j.1365-2929.2004.01819.x]

9. University of the Free State (UFS). Community service policy of the University of the Free State. 2006. http:// University of the Free State (UFS). Community service policy of the University of the Free Sta
supportservices.ufs.ac.za/dl/userfiles/Documents/00000/357_eng.pdf (accessed 26 August 2015).

10. Marzano RJ. Dimension 1: Positive Attitudes and Perceptions About Learning. A Different Kind of Classroom: Teaching with Dimensions of Learning. Alexandria, VA: Association for Supervision and Curriculum Development, 1992. http://files.eric.ed.gov/fulltext/ED350086.pdf (accessed 26 August 2015).

11. Higher Education Quality Committee (HEQC). Service-Learning in the Curriculum: A Resource for Higher Education Institutions. Pretoria: Council on Higher Education, 2006.

12. Morgan S, Smedts A, Campbell N, Sager R, Lowe M, Strasser S. From the bush to the big smoke - development of a hybrid urban community based medical education program in the Northern Territory, Australia. Rural Remote Health 2009;9(3):1175. 